\title{
Phenotypic Constraints and Phenotypic Hitchhiking in a Promiscuous Enzyme
}

\author{
Andreas Wagner ${ }^{1,2,3, *}$ and Christian Weikert ${ }^{1}$ \\ ${ }^{1}$ Institute of Evolutionary Biology and Environmental Studies, University of Zurich, CH-8057 Zurich, Switzerland \\ ${ }^{2}$ The Santa Fe Institute, Santa Fe, New Mexico 87501, USA \\ ${ }^{3}$ Swiss Institute of Bioinformatics, CH-1015 Lausanne, Switzerland
}

\begin{abstract}
Covarying phenotypic traits can limit natural selection's ability to modify these traits. Most evolutionary studies on trait covariation use the comparative method to study complex traits of multicellular organisms. Simpler traits have the advantage of being amenable to experimental evolution. Here we study such a simple molecular system, the TEM-1 beta-lactamase protein, a promiscuous enzyme that hydrolyses antibiotics, and thus confers antibiotic resistance to bacteria. We mutagenized large populations of TEM-1, and selected in these populations for activity on the four different antibiotics ampicillin, carbenicillin, oxacillin, and penicillin G. While mutagenesis alone lead to highly correlated changes in enzyme activity on the four antibiotics, subsequent selection was able to modify this activity covariation substantially, and even lead to uncorrelated activities. We found that selection on one antibiotic A may be more effective in increasing activity on another antibiotic B then selection on antibiotic B itself. We call this phenomenon phenotypic hitchhiking. It can be readily explained through quantitative genetic theory as a consequence of differing variances in covarying traits. It may help engineer macromolecules with desirable properties through directed evolution. Our analysis is a first step towards systematic analysis of trait covariation in experimental molecular systems. It shows that selection may readily modify the phenotypic constraints that limit its efficacy.
\end{abstract}

Keywords: Lactamase, antibiotic resistance, laboratory evolution, trade-offs.

\section{INTRODUCTION}

An organism or genotype shows phenotypic plasticity if it can form more than one phenotype depending on its environment. Phenotypic plasticity is ubiquitous, and occurs on all levels of biological organization, from visible, macroscopic phenotypes down to the conformations of molecules [1-17]. Historically, most studies of plasticity have focused on macroscopic or physiological traits [1]. However, in recent years, evidence for the plastic traits of individual molecules has been mounting. Such plasticity is usually called by different names, such as cross-reactivity, substrate ambiguity, moonlighting, or promiscuity [15-19]. The phenotype in question is a spatial fold (conformation) of a molecule that changes in time and permits the molecule to interact with different other molecules. Promiscuity, for example, is a property of many enzymes whose catalytic abilities are not highly specific to one substrate molecule. Instead, such enzymes can catalyze reactions with different substrate molecules $[15,16,18]$. Promiscuity is an instance of phenotypic plasticity, if one considers that any one enzyme molecule exists in continually fluctuating molecular environments, for example through the thermal motions of molecules in its

*Address correspondence to this author at the Institute of Evolutionary Biology and Environmental Studies, University of Zurich, CH-8057 Zurich, Switzerland; Tel: +41-635-6141; Fax: +41-635-6144;

E-mail: andreas.wagner@ieu.uzh.ch vicinity. In consequence, its conformation changes incessantly, thus also altering its ability to react with any one substrate molecule.

How phenotypes (plastic or not) change in response to natural selection is among the most central questions of evolutionary biology. Complex phenotypes are not univariate variable along one scalar axis - but multivariate. Examples include the shapes of vertebrate skulls and jaws, whose description involves dozens of morphometric landmarks, cuticular hydrocarbon composition of plants, or an individual's fitness itself, which can be decomposed into multiple scalar life history traits, such as survivorship or fecundity [20-22]. The individual scalar traits that compose such complex phenotypes typically do not vary independently, but show covariation within a population. If natural selection changes one trait, correlated changes in others often occur. The causes and consequences of such correlated changes often referred to as phenotypic constraints - are the subject of an enormous body of literature [21, 23-36].

One can distinguish two fundamental kinds of correlated changes in quantitative traits. In the first, an increase in some trait $X$ on which natural selection acts directly causes a correlated increase in some other trait Y. In other words, the two traits covary positively. In the second kind of correlated change, an increase in trait $\mathrm{X}$ causes a decrease in trait $\mathrm{Y}$. In this case, the two traits $\mathrm{X}$ and $\mathrm{Y}$ covary negatively, which may reflect a trade-off between the traits. Trade-offs are fre- 
quent from molecular to organismal phenotypes, and especially in life-history traits [22, 37-42].

In both kinds of correlated change, phenotypic evolution is constrained by trait covariance. That is, natural selection can change several covarying traits in some directions but not in others, at least on short evolutionary timescales. On longer evolutionary timescales, this covariance itself can change, such that traits that show strong positive covariance in one species, may covary less, not at all, or even negatively in another species [20, 21, 27-29, 43-47]. For multiple univariate phenotypic traits $P_{1}, \ldots, P_{\mathrm{n}}$ trait covariance is usually described by a phenotypic covariance matrix $\mathbf{P}$ whose entries describe the covariance between traits $P_{i}$ and $P_{j}$. Closely related to this matrix is the genetic covariance matrix G, which describes phenotypic covariation that is due to genotypic differences among organisms. The structure of $\mathbf{G}$ is much more difficult to estimate, which is why most pertinent studies focus on phenotypic covariation [27].

Trait covariation is well studied for macroscopic phenotypes, but much less so for molecular phenotypes. Here we analyze trait covariances and correlations for the promiscuous activity of an enzyme, where trait $\mathrm{P}_{i}$ corresponds to the enzyme's catalytic activity on some substrate i. While promiscuous activities have been studied in many enzymes [15$18,48,49]$, there is very little systematic work on how different promiscuous activities covary. However, such covariance could be quite important to understand the evolution of protein functions and its limits, a topic of interest not only to evolutionary biologists but also to protein engineers $[17,18]$. Positive covariance of a protein's activity on different substrates means that one cannot increase activity on one substrate, without also increasing activity on another substrate. If the second substrate is an undesirable byproduct of a reaction, this covariation would need to be reduced before engineering a protein with high specificity to the first substrate. Conversely, enzymes may face trade-offs between activity on different substrates, where an enzyme highly active on one substrate would have little activity on other substrates Candidate examples are reviewed in [18]. In such enzymes, simultaneous high activity on multiple substrates might be difficult to engineer or evolve. Although one might think that such trade-offs must be very frequent, some studies fail to show them [18, 49]. A case in point is a recent laboratory evolution study on paraoxonase, bacterial phosphotriesterase, and carbonic anhydrase, three different promiscuous enzymes. This study identified mutations that increased the enzymes' activity on a minor, promiscuous substrate, while affecting activity on the major substrate little, or even increasing this activity [49]. Studies like this typically compare only few (as few as two) variants of a protein [18], which is insufficient to ascertain a trade-off or any other pattern of covariation with much confidence. For that purpose, one needs to study trait covariation more systematically and for larger samples.

We here provide a first step towards marrying wellestablished methods in evolutionary genetics with laboratory evolution of protein molecules. We do so for a paradigmatic example of a promiscuous enzyme, the $\beta$-lactamase TEM-1. $\beta$-lactamases are enzymes that hydrolyze antibiotics with a four carbon ring structure called a $\beta$-lactam. This class of antibiotics includes prominent molecules, such as penicillin and ampicillin $[50,51]$. $\beta$-lactam antibiotics interfere with proteins required for synthesizing the bacterial cell wall. Through their ability to inactivate these antibiotics, $\beta$ lactamases are a major cause of bacterial resistance against antibiotics. We randomly mutagenized DNA molecules encoding TEM-1, and subjected four separate populations of $E$. coli cells expressing these molecules to selection for resistance against the four antibiotics ampicillin (A), carbenicillin (C ), oxacillin (O), and penicillin G (P). From each of the resulting four populations, we isolated 48 clones. We then measured the hydrolytic activity of each TEM-1 clone on all four antibiotics, and studied the change in this activity individually, as well as for all pairs of antibiotics, through their phenotypic covariance matrix $\mathbf{P}$. We find that selection can change the pattern of activity covariation rapidly, and allows for a phenomenon that we call phenotypic hitchhiking, which can be explained through basic quantitative genetic theory.

\section{MATERIALS AND METHODS}

\section{Vector Construction and Host Strain Transformation}

The starting point of our work is the class A serine $\beta$ lactamase TEM-1, a well studied enzyme [50-52]. Its coding DNA sequence can be found in the European Nucleotide Archive (Database ID AP010961.1 at http://www.ebi.ac.uk/ ena/) . We had a DNA fragment containing the TEM-1 coding sequence synthesized by GENEART (Regensburg, Germany). The fragment comprises position 114462 to 113652 of the above database entry.

Our cloning vector is a derivative of the low copy number plasmid pUA66 [53], which we obtained by placing a cassette comprising the lac-promoter and a multiple cloning site from plasmid pSK (Stratagene, La Jolla CA, USA) upstream of the EGFP gene in pUA66. We obtained this cassette through polymerase chain reaction (PCR) amplification of pSK, using the primer LacBX (39bp: 5' CGGAA GATCT CGAGC GTTTA CACTT TATGC TTCCG GCTC 3’) upstream of the lac promoter with respect to the multiple cloning site, and the bacteriophage T7-primer (20bp: 5' TAATA CGACT CACTA TAGGG 3') heading in the opposite direction, and cloned the amplified fragment into the BglII and Xho sites of plasmid PUA66. The important features of this plasmid are a gene encoding resistance to the antibiotic kanamycin, which we used to select for the presence of the vector in transformed cells, as well as a lac promoter, which drives the expression of a DNA insert. We used E.coli strain $\mathrm{Dh} 5 \alpha$ as a cloning host, which confers expression of the lac promoter even in the absence of an inducer [54]. We expressed the inserted gene without adding inducer during our selection experiments, in order to enable selection using low antibiotic concentrations and, more importantly, to alleviate previously demonstrated selection against high levelexpression of our focal proteins [54].

To clone the TEM-1 DNA into the cloning vector, we first digested the vector with the restriction enzymes SmaI and EcoRI, which creates one blunt end and one overhanging end. We then PCR-amplified the TEM-1 DNA using the primers P1 and P2 (P1: 5'GG AGC TTA TGA GTA TTC AAC ATT TTC GTG 3', P2: 5' AA TTG GAT CCT CAT CCA TAG TTG CCT GAC3'), which introduce restriction sites for the enzyme EcoRI at their end. We then took advantage of the 3'-5' exonuclase activity of the Klenow frag- 
ment of DNA polymerase to render one of the amplified DNA's ends blunt-ended, while leaving the other end complementary to an EcoRI restriction site on the 3' end. We used this procedure because preliminary experiments (not shown) had demonstrated that this procedure was more than 50 times more efficient than blunt end cloning. Specifically, we added the Klenow fragment to the PCR-amplified insert in a $20 \mu \mathrm{l}$ reaction volume using $0.2 \mathrm{U} / \mathrm{ml}$ Klenow enzyme in NEB Buffer 2 (both NEB, New England Biolabs, Ipswich MA, USA) supplemented with dCTP at $50 \mathrm{uM}$. The reaction proceeded at $37^{\circ} \mathrm{C}$ for 30 minutes. We ligated the products of this reaction into the cloning vector in a $20 \mu \mathrm{l}$ reaction volume, using a cyclic temperature change regime (10 minutes at $30^{\circ} \mathrm{C}$ followed by 10 minutes at $10^{\circ} \mathrm{C}$, repeated 48 times), after which we heat-inactivated the ligation reaction $60^{\circ} \mathrm{C}$ for $20 \mathrm{~min}$.

We used the following procedure (modified from [55, $56])$ to prepare chemically transformation-competent $E$. coli cells of strain Dh5 $\alpha$. Briefly, after growth at $20^{\circ} \mathrm{C}$ to an optical density (OD) 600 of 0.3 , using SOB medium( $0.5 \% \mathrm{w} / \mathrm{v}$ yeast extract, $2 \% \mathrm{w} / \mathrm{v}$ tryptone, $10 \mathrm{mM} \mathrm{NaCl}, 2.5 \mathrm{mM} \mathrm{KCl}$, and $20 \mathrm{mM} \mathrm{MgSO} 4)$, we collected cells by centrifugation (3000xg, 10 minutes, $4^{\circ} \mathrm{C}$ ). After resuspension in $40 \%$ of the original culture volume in buffer CCMB80 (10 mM KOAc pH 7.0, $80 \mathrm{mM} \mathrm{MnCl2,10mM} \mathrm{MgCl2,} \mathrm{10 \%} \mathrm{v/v} \mathrm{glycerol,}$ $\mathrm{pH}$ adjusted to 6.4 if required using $0.1 \mathrm{~N} \mathrm{HCl}$, sterile filtrated and kept at $4^{\circ} \mathrm{C}$ ), cells were kept on ice for 20 minutes. Following another collection using centrifugation under the same conditions, we resuspended cells again in CCMB80 buffer, but now in 4 percent of the initial culture volume.

After another incubation on ice for 20 min, we used cells for transformation either immediately or aliquoted them and stored them at $-80^{\circ} \mathrm{C}$ for later use. We used 100 ul of competent cells for transformation under the following protocol. We briefly thawed aliquots of chemically competent cells on ice, and added $10 \mu \mathrm{l}$ of a ligation reaction. We incubated the cell suspension for 30 minutes on ice, heat-shocked it for 60 minutes in a water-bath at $42^{\circ} \mathrm{C}$, and added $900 \mu \mathrm{l}$ SOB supplemented with glucose to $20 \mathrm{mM}$. After recovery of the cells for 90 minutes under vigorous agitation at $37^{\circ} \mathrm{C}$, which enables expression of the kanamycin resistance gene of the plasmid, we plated 50ul of transformed cells on LB plates containing kanamycin at $30 \mu \mathrm{g} / \mathrm{ml}$ to select for successful transformation. We continued further experimentation with transformed cells only if at least 1000 clones were obtained on this reference plate.

\section{PCR Mutagenesis}

For PCR-based mutagenesis, we used the same primer pair P1 and P2 described above, and the Taq-Polymerase (2.5 U/ reaction),(NEB)) under the following reaction conditions:20 ng template, $0.2 \mathrm{mM}$ dGTP, $0.2 \mathrm{mM}$ dATP, $1.0 \mathrm{mM}$ dCTP, $1.0 \mathrm{mM}$ dTTP with standard Taq-buffer, supplemented with $1.5 \mathrm{mM} \mathrm{MgCl} 2,1.5 \mathrm{mM} \mathrm{MnCl} 2$, and $2.5 \mathrm{U}$ Taq in a $100 \mu$ reaction volume. The cycling protocol comprised 25 cycles (each $94^{\circ} \mathrm{C}$ for $1 \mathrm{~min}, 45^{\circ} \mathrm{C}$ for $1 \mathrm{~min}$ and $72^{\circ} \mathrm{C}$ for 1 min). Based on previous observations [57] this procedure introduces an expected number of approximately 0.0066 nucleotide changes per nucleotide and PCR reaction, which yields an expected number of $0.0066 \times 81 \& 5.3$ mutations for the 810 base pair long amplified TEM-1 coding sequence.
This mutagenic PCR protocol has only a mild bias towards mutations away from $\mathrm{T}$ and towards $\mathrm{A}$ [57]. We cloned the reaction products into the cloning vector using the protocol we described above under vector construction. We estimate that the mutagenized population comprises $1.5 \times 10^{10}$ TEM- 1 molecules, an assertion that is based on the $20 \mathrm{ng}$ purified DNA used for the ligation reaction, a fragment length of 827 bp, as well as the fact that $1 \mathrm{~kb}$ of DNA comprises approximately $9.1 \times 10^{11}$ molecules per $\mu$ g. Under our reaction conditions, and under the assumption that mutations follow a Poisson distribution, we expect only a tiny fraction of exp($5.3) \approx 0.005$ of sequences to have experienced no mutation.

\section{Cultivation Conditions and Selection}

Immediately after transformation of cells with ligation products as described above, we plated a small aliquot on solid LB-medium (Becton Dickinson, 1.5\% agarose) containing kanamycin alone to estimate the absolute number of clones entering selection for each of the four focal antibiotics. We distributed the remaining volume on plates with the same medium supplemented with individual antibiotics at two different concentrations, as specified below, and incubated the plates over night at $37^{\circ} \mathrm{C}$. In order to standardize the cultivation conditions, we prepared all plates fresh on the day of the experiment. We added the agar to solid antibiotics and used a total agar volume of $15 \mathrm{ml}$ for each $100 \mathrm{~mm}$ petri dish. After overnight cultivation, a plate needed to show sufficiently many colonies for isolation of multiple clones (see below), yet not too many to prevent picking of individual colonies. To achieve the best colony density, we explored two different antibiotic concentrations, as we describe now. For selection on ampicillin, we plated approximately $2.7 \times 10^{4}$ cells on two plates containing 200 and $300 \mu \mathrm{g} / \mathrm{ml}$ of ampicillin, and picked colonies for further analysis from the latter plate. For selection on carbenicillin, we plated approximately $2.5 \times 10^{4}$ cells on two plates containing 250 and $375 \mu \mathrm{g} / \mathrm{ml}$ of carbenicillin, and picked colonies for further analysis from the latter plate. For selection on oxacillin, we plated approximately $2.3 \times 10^{4}$ cells on two plates containing 250 and 375 $\mu \mathrm{g} / \mathrm{ml}$ of oxacillin, and picked colonies for further analysis from the latter plate. For selection on penicillin $G$, we plated approximately $2.1 \times 10^{4}$ cells on two plates containing 200 and $300 \mu \mathrm{g} / \mathrm{ml}$ of penicillin $\mathrm{G}$, and picked colonies for further analysis from the latter plate. Under these conditions, we usually observed 200-500 colonies per plate. We picked multiple clones from each plate (see below), and washed the remaining colonies off the plate using LB medium to create glycerol stocks.

We also explored the effects of mutagenesis in the absence of selection. To this end, we plated $50 \mu \mathrm{l}$ of a transformation mixture on a plate containing only kanamycin at $30 \mu \mathrm{g} / \mathrm{ml}$, and incubated the plate over night at $37^{\circ} \mathrm{C}$.

\section{Clone Isolation}

From each of the five plates thus described, we picked 54 colonies, and streaked them on LB-plates containing kanamycin at $30 \mu \mathrm{g} / \mathrm{ml}$ (and no further antibiotics). We chose this 
number of clones to enable the preparation of about 48 cell extracts for activity measurements, the excess accounting for the possibility that some picked clones may fail to form colonies after transfer from the primary plate. Each clone was assigned an individual number.

\section{Periplasmic Extracts}

From each clone, we inoculated a pre-culture in one well of a 96-well plate, with a culture-volume of $150 \mu$ l using LBmedium with kanamycin at $30 \mu \mathrm{g} / \mathrm{ml}$. This inoculum was allowed to grow for 6 to 8 hours at $37^{\circ} \mathrm{C}$ on an incubatorshaker at $650 \mathrm{rpm}$ ( VWR, model $980141 \mathrm{CH}$ ). We then used $20 \mu \mathrm{l}$ of this preculture to inoculate a 96 deep-well plate (Sarstedt, 82.1972.002) with a culture volume of $1.5 \mathrm{ml}$ containing one glass bead ( $4 \mathrm{~mm}$, Merck) per well, using the same medium as for the pre-culture, but supplemented with $200 \mu \mathrm{M}$ IPTG, to increase beta-lactamase expression, and thus allow for activity measurement of enzymes with low specific activities.

After growth overnight $\left(37^{\circ} \mathrm{C}, 650 \mathrm{rpm}\right)$ we prepared periplasmic extracts using a modification of a protocol described by [58]. Briefly, we collected cells by centrifugation for 20 minutes at $2250 \mathrm{xg}$ at $4^{\circ} \mathrm{C}$. We removed the supernatant and resuspended the pellets in $100 \mu \mathrm{l}$ chilled extraction buffer (0.2M Na-Acetate, $\mathrm{pH} 5.9$, on ice). The glass beads we had used during growth in the deep-well plates facilitated resuspending the cell pellets with a vortex mixer. We then conserved the cells prior to extraction by transferring them to 96-well Multiply PCR-plates (Sarstedt, Nümbrecht, Germany, 72.1978.202) sealed with sealing-tape (Sarstedt, 95.1994), and by freezing these plates at $-20^{\circ} \mathrm{C}$ for further processing. We next describe how to prepare the periplasmatic fraction. We briefly thawed plates containing the cell suspensions at ambient temperature, and followed this treatment by four-freeze thaw cycles. Each of these cycles proceeded as follows: brief immersion of the plate into liquid nitrogen until the sizzling sound of boiling nitrogen ceases, incubation of plates in a water bath $(10$ minutes at $37^{\circ} \mathrm{C}$ ) homogenization of content of the wells by vortexing. After these cycles we added another $100 \mu$ l extraction buffer to the wells, and kept the extracts on ice for 30 minutes. Subsequently, we clarified the extracts by centrifugation (30 minutes, $\left.13000 \mathrm{xg}, 4^{\circ} \mathrm{C}\right)$. We transferred the supernatant for each culture to $1.5 \mathrm{ml}$ Sarstedt screw-cap-tubes (Sarstedt, 72.692) and stored it at $-20^{\circ} \mathrm{C}$ for enzymatic analysis.

\section{Enzyme Activity Measurements}

We used microiodometry to determine the hydrolytic activity of different TEM-1 variants on the four antibiotics we studied. In this method, a starch iodine complex is decolorized by penicilloic acid, which is formed by the action of $\beta$-lactamase on penicillins [59]. For the starch solution, we prepared hydrolyzed starch using a suspension of $5 \%$ soluble starch, which we boiled to form a viscous solution. To this solution we added concentrated hydrochloric acid to a final concentration of $10 \mathrm{mM}$, and continued boiling for 10 minutes. To quench starch hydrolysis, we chilled the reaction while stirring it on ice, and neutralized it using sodium carbonate. To prepare the iodine-starch reagent used in the assay, we supplemented $50 \mathrm{mM}$ potassium phosphate ( $\mathrm{pH}$ 5.9) with starch solution to a $0.5 \%$ final concentration, and to 270 $\mu \mathrm{M}$ in iodine using an iodine stock solution (80mM I2 /
3.2M KI). For activity measurements, we supplemented 50 $\mathrm{mM}$ potassium phosphate with the iodine-starch reagent to a final starch concentration of $0.16 \%$ and with the individual $\beta$-lactam antibiotic to $400 \mu \mathrm{M}$ in each well.

In order to standardize the measurements, we thawed the Sarstedt-tubes containing periplasmic extracts (see above) briefly using an ice-bath, centrifuged them (15000xg, 20 minutes, $4^{\circ} \mathrm{C}$ ), and kept them on ice throughout the analysis. Furthermore, we let the mixtures containing the iodine/starch reagent and the individual substrates equilibrate for 20 minutes at room temperature prior to transfer to 96-well plates (Sarstedt, 95.1994) for measurement, as we describe next.

To each well of a 96-well plate we added $175 \mu$ l reagent, which we supplemented with $10 \mu$ l diluted periplasmic extract with the aid of a 8-channel multipipettor (BioHit, Helsinki, Finland). Specifically, we diluted the extract 1:1 for measurement of activity on carbenicillin and oxacillin, and 1:9 for measurement of activity on penicillin $G$ and ampicillin as substrates, using $50 \mathrm{mM}$ potassium phosphate $(\mathrm{pH}$ 5.9) supplemented with a trace of BSA.

We followed the absorption of the reaction mix at 600 nm using a microplate-reader (Infinite F200 Pro, TECAN, Männedorf, Switzerland), which was temperature controlled at $30^{\circ} \mathrm{C}$, and was equipped with a $600 \mathrm{~nm}$ filter. We set the time interval between successive reads from a single well to 80 s, and used linear regression of the absorptions over time for individual wells to estimate the reaction-rates. In each 96 well plate, we also used blank reactions comprising samplebuffer and extraction-buffer in the same ratios as used for the measurements of the extracts, in order to estimate background reaction rates arising from the spontaneous decomposition of substrates. We measured all extracts in duplicate (in different wells), and included only data in our analysis where the duplicate measurements deviated from each other by less than 15 percent.

To determine the activity of the ancestral TEM-1 clone on the four different antibiotics, we obtained single colonies, and prepared inocula, cultures, and extracts as described for the mutagenized/selected populations above.

The resulting measurements yield information about the activity of $\beta$-lactamases expressed per unit volume (mODU $\left.\left(\min ^{-1}\right)(10 \mu \mathrm{l} \mathrm{extract})^{-1}\right)$, i.e., the volumetric activity, which meets our requirements, as we are mainly interested in relative activities among different clones, not in establishing absolute activity values.

\section{Statistical Analysis of Covariance Matrices}

To compare correlation or covariance matrices is nontrivial, because any two such matrices could differ in more than one way. Multiple methods to compare correlation or covariance matrices exist [21, 23, 25, 27, 60-63]. Among them, we chose common principal component analysis (CPCA), because it allows one to compare different aspects of matrix structure [60, 64]. It is built on principal component analysis, a multivariate statistical technique that aims to identify directions in a set of multivariate data (a cloud of points in an $n$-dimensional space) along which most of the variation in the data occurs [65]. Principal component analysis computes a new coordinate system in this space, where the largest amount of variation in the original quantity occurs 
along the first axis (the first principal component), the second largest amount along the second principal component, and so on. These principal components are the eigenvectors of the covariance matrix of the data, sorted by descending magnitude of the eigenvalue associated with each eigenvector. Principal component analysis is usually used to reduce the complexity of high-dimensional data sets, but it can also serve as a starting point to compare the structure of two or more covariance matrices, that is, for CPCA. CPCA is based on the so-called Flury hierarchy of shared principal components between covariance matrices [64]. It starts with the observation that two covariance matrices can be either exactly equal, or they can differ in multiple different ways. First, and most trivially, the entries of two matrices may differ by a single multiplicative constant. Second, two matrices may have identical principal components but different eigenvalues. Third, they may differ in one of their principal components. Fourth, they may differ in two, three, or more principal components, up to $n-2$ principal components. Because principal components are by definition orthogonal to one another, the first $n-1$ principal components completely determine the remaining principal component. Thus, matrices that differ in all $n-1$ principal components are completely unrelated to one another. These different levels of matrix similarity are hierarchical, in the sense that matrices which share, say, three principal components, also automatically share two different principal components. CPCA tests the above null-hypotheses of matrix similarity for two or more covariance matrices. We used the software срс [http://pages.uoregon.edu/pphil/software.html, 60] in all such hypothesis testing. We note that CPCA is designed for covariance and not correlation matrices.

\section{RESULTS}

\section{Different Selection Regimes Show Different Average Trait Outcomes}

We first created a population of approximately $1.5 \times 10^{10}$ TEM-1 variants using PCR mutagenesis (see Methods), and transformed $E$. coli cells with an expression vector that encoded these variants. For each of our focal antibiotics (P,A,C,O) we then plated between 20,000 and 30,000 transformants on agar plates with solid LB medium that con- tained the antibiotic for selection. From the surviving colonies, we selected 48 clones, prepared periplasmic extracts from them, and measured the volumetric hydrolytic activity - referred to below simply as activity - of the $\beta$-lactamase they encode. Importantly, we measured the activity of each clone on all four different antibiotics. In addition, we also measured the activity of 48 mutagenized variants that had not been subject to selection on any of the four antibiotics (Fig. 1). All activity measurements we report use volumetric units of Optical Density change [mODU $\left(\mathrm{min}^{-1}\right)(10 \mu \mathrm{l} \mathrm{ex}-$ tract) ${ }^{-1}$.

Two processes with opposing effect influence the hydrolytic activities of the $\beta$-lactamases in the population that survives selection. The first is mutation, which tends to reduce the average activity relative to the wild type ancestor. The second is selection, which tends to increase the average activity, because it eliminates clones with low activity. When mutation and selection interact, three qualitatively different outcomes are possible. The first occurs if mutagenesis created few or no molecules with higher activity than the ancestral molecule. Because mutation reduces activity on average [66-69], this means that the average activity of survivors will also be reduced. In the second outcome, the effects of both processes balance each other, such that no change in the average activity relative to the ancestor occurs. In the third outcome, mutagenesis creates many variants that confer higher activity than the ancestor, leading to an increased average activity of the survivors.

Our experiments show instances of the first scenario (for carbenicillin), the second scenario (oxacillin and penicillin $\mathrm{G}$ ), and the third scenario (ampicillin, Fig. 2). Specifically, on carbenicillin, mutagenesis dramatically reduced activity to $13.8( \pm 5.9$ s.dev.) compared to $123.6 \pm 6.9$ in the ancestor (a difference significant at $p=0.001$, Mann-Whitney U-test). Selection increased activity more than four-fold to $64.3 \pm 21.2$, but did not recover the ancestral activity fully ( $p=0.0017$, Mann-Whitney U-test, Fig. 2A).The mean activity of the TEM-1 ancestor on oxacillin was equal to $43.9 \pm 7.3$ , whereas after mutagenesis, this activity reduced to 21.15 \pm 16.7 , which is statistically significantly lower $(p=0.02$, Mann-Whitney U-test ). After the subsequent selection step, this activity recovered to a level statistically indistinguisha-

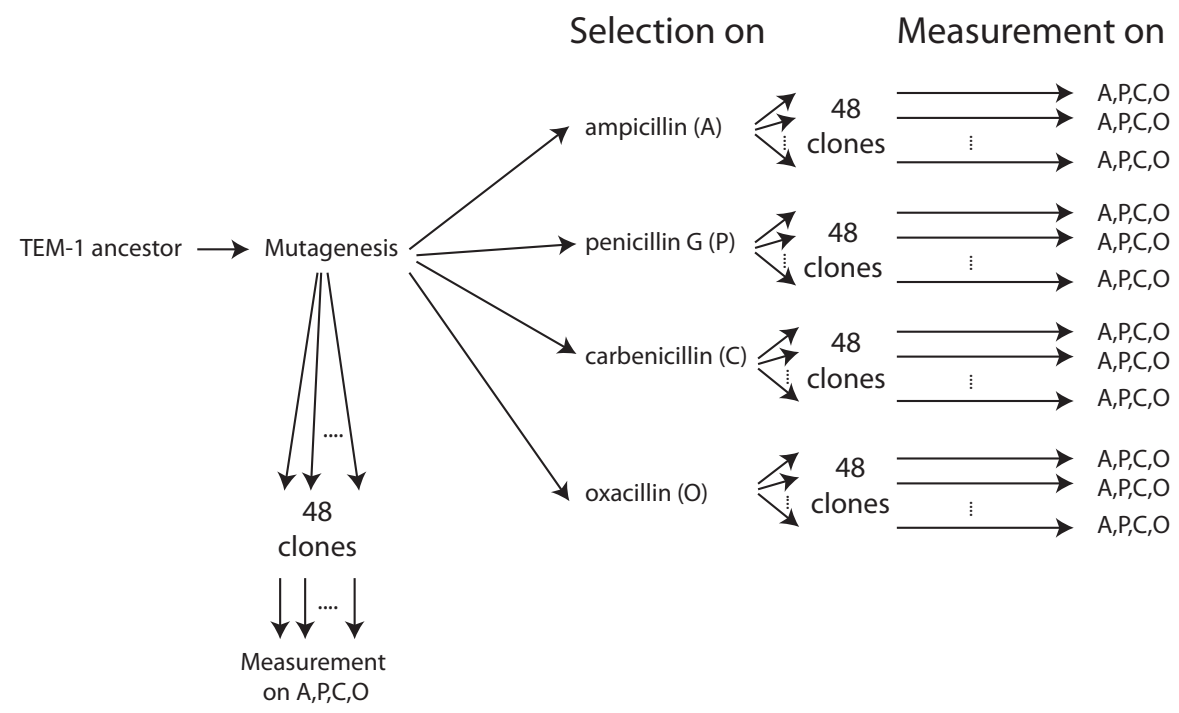

Fig. (1). The experimental design used in this study. 

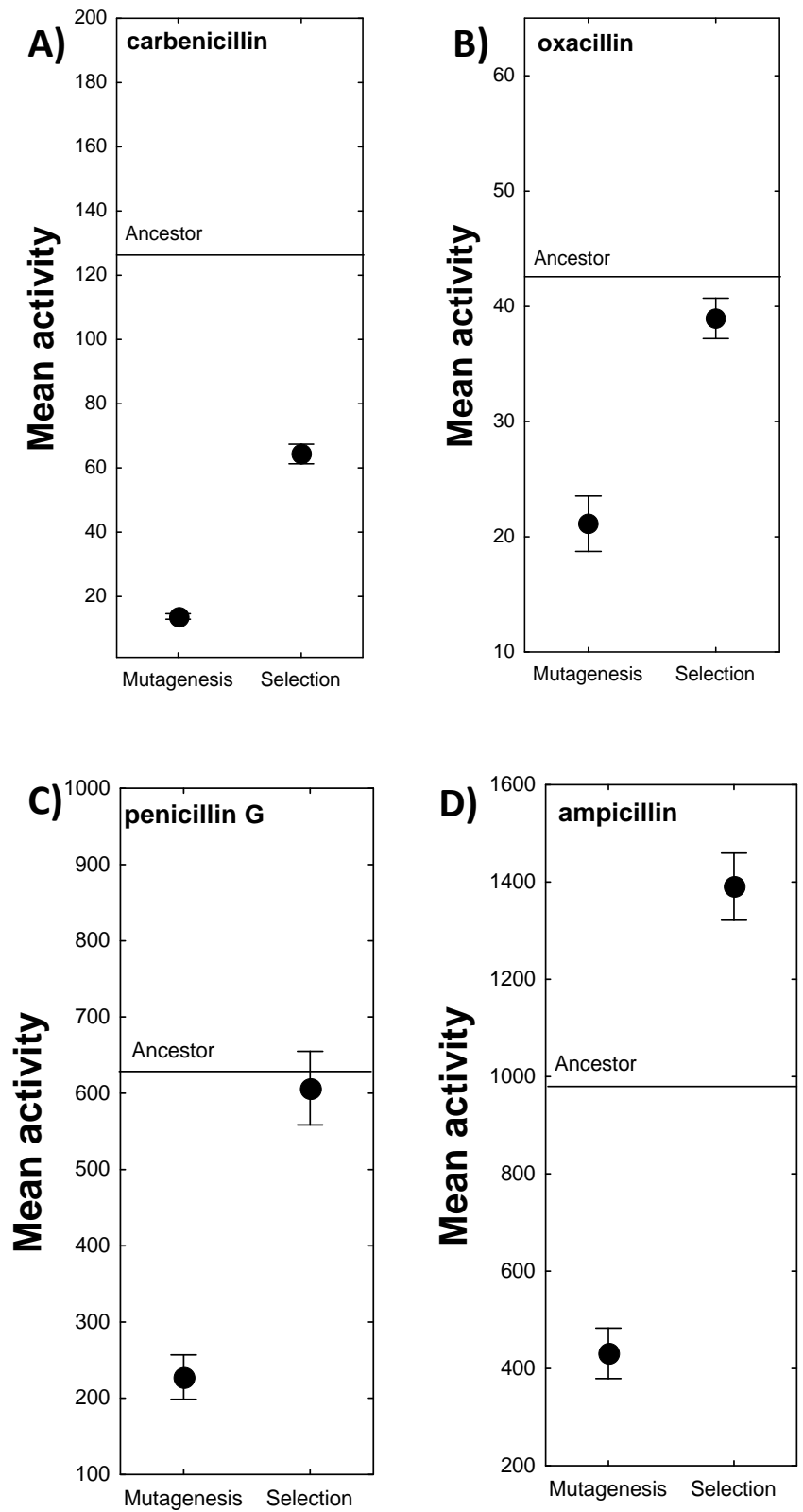

Fig. (2). Activity changes of TEM-1 $\beta$-lactamases after mutagenesis and selection on four different antibiotics. The vertical axes show means (dots) and one standard error of the mean (bars) in activity of 48 clones of TEM-1 $\beta$-lactamase on the antibiotic indicated in the inset. The horizontal axes indicate whether the clones stem from populations that had only been subject to mutagenesis (left) or to mutagenesis followed by selection (right) on $\mathbf{A}$ ) carbenicillin, B), oxacillin, C) penicillin G, and D) ampicillin. Horizontal lines indicate the mean activity of the TEM-1 ancestor on each antibiotic. Units are mODU $\left(\mathrm{min}^{-1}\right)(10 \mu \mathrm{l} \text { extract })^{-1}$.

ble from that of the ancestor (39 $\pm 12, p=0.36$, Mann-Whitney U-test, Fig. 2B). On penicillin G, mutagenesis reduced activity significantly from $624.2 \pm 44.5$ in the ancestor to $227.9 \pm 202$ in the mutagenized population ( $p=0.003$, MannWhitney U-test), and selection increased the activity to a level that is indistinguishable from the ancestor (606.8 \pm 335 , $p=0.19$, Fig. 2C). Finally, on ampicillin, the ancestor showed an activity of $985.7 \pm 36.8$, which mutagenesis reduced signif- icantly by more than 50 percent to $431 \pm 360.1 \quad(p=0.01$, Mann-Whitney U-test). The subsequent selection step increased mean activity significiantly $(p=0.015$, MannWhitney U-test ) to approximately 40 percent above that of the ancestor (1390.4 \pm 478.1 , Fig. 2D). In sum, mutagenesis significantly reduced activity on all four antibiotics, a reduction for which subsequent selection could partially or fully compensate (Fig. 2).

\section{Activities on Different Antibiotics Covary Positively}

We next asked to what extent the activities on different antibiotics are positively correlated, negatively correlated, or uncorrelated. We first focused on 48 clones that had been subjected to mutagenesis only, without subsequent selection on any antibiotic. Fig. (3) shows the result in the form of 7 pairwise scatterplots. All pairwise activities are highly positively correlated, with a Pearson correlation coefficient $r>0.96$ that is highly significantly different from zero $\left(p<10^{-17}\right)$. The matrix in Fig. (5A) summarizes these Pearson correlation coefficients below the diagonal in black type. (Diagonal and upper diagonal entries correspond to phenotypic variances and covariances of these traits.) This observation shows that mutation alone induces highly correlated changes in activities on different antibiotics. It suggests that the effects of most mutations are not specific to one antibiotic, but reduce activity on all antibiotics in a similar way.

We next asked the same question for all 48 clones isolated from each selection line. Fig. (4) shows, as an example, pairwise scatterplots of these activities for the population selected on ampicillin, where selection increased activity to the greatest extent. The data shows that some activities are highly correlated in this population, for example activity on ampicillin and on penicillin $\mathrm{G}$ (top left panel, $\mathrm{r}=0.89, p<10^{-3}$ ). Others are more weakly correlated, for example, activity on oxacillin and on penicillin $\mathrm{G}$ (bottom left panel, $\mathrm{r}=0.38$, $p<0.008$ ). The matrix in Fig. (5B) shows below its diagonal the pairwise Pearson correlation coefficients for this population. It indicates that four of the seven pairwise correlation are positive, whereas two (C-P, C-A) are not significantly different from zero. Note that not a single correlation is significantly negative.

The lower-diagonal matrices Fig. (5C) through 5E summarize the same data for the three populations that we subjected to selection on carbenicillin, oxacillin, and penicillin $\mathrm{G}$. The data show that the nature of the selection influences the extent to which individual activities are correlated. For example, after selection on oxacillin only two pairs of activities show a significantly positive association, whereas after selection on penicillin $G$ (Fig. $5 E$ ), all pairs show not only a significant association, but this association exceeds $r=0.86$ for every single pair.

In sum, mutagenesis alone introduces highly correlated changes in activity on individual antibiotics. These correlations can be substantially weakened by selection. Their magnitude depends on the kind of selection applied to a population. Not a single pair of antibiotics exhibits the significantly negative association of activity that might be indicative of a trade-off.

Visual inspection of matrix entries in Fig. (5) can reveal differences in how activities on pairs of antibiotics covary, but it does not answer how similar or different the matrices 

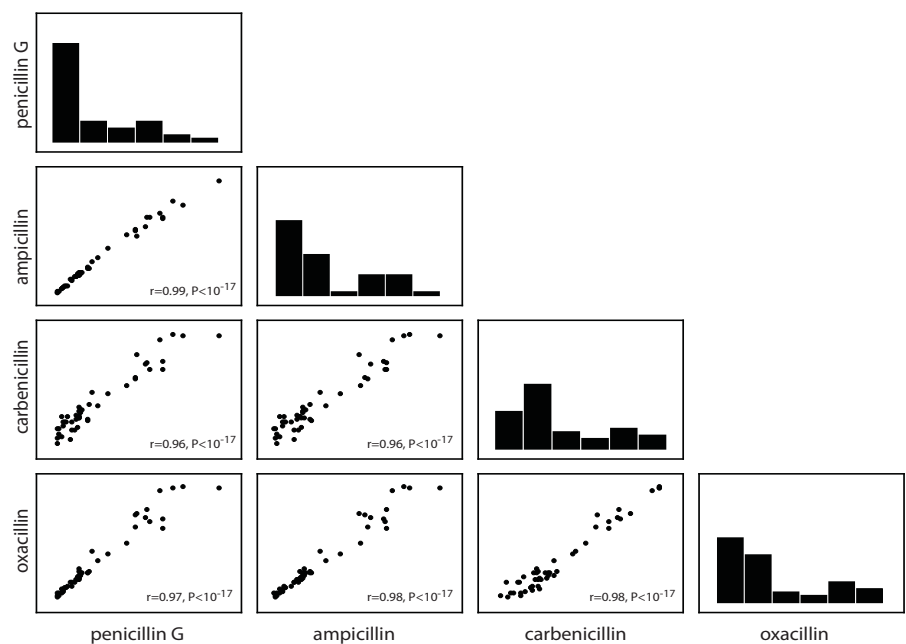

Fig. (3). Covariation of activity on four antibiotics after mutagenesis. All data in this figure are based on 48 clones from TEM-1 populations subject to mutagenesis but no subsequent selection (Fig. 1). The four histograms in the diagonal of the lower-diagonal matrix indicate, from top to bottom, the distribution of activities of these clones on penicillin G, ampicillin, carbenicillin, and oxacillin. The scatterplots indicate activities on pairs of antibiotics, as indicated to the left of each row and on the bottom of each column. Axes scales are omitted for clarity and correspond to the following intervals bounded by minimal and maximal measured activity values: $(25.8$, 839.0) on penicillin G, (48.9, 1418.6) on ampicillin, $(6.0,27.0)$ on carbenicillin, $(3.7,58.2)$ on oxacillin.
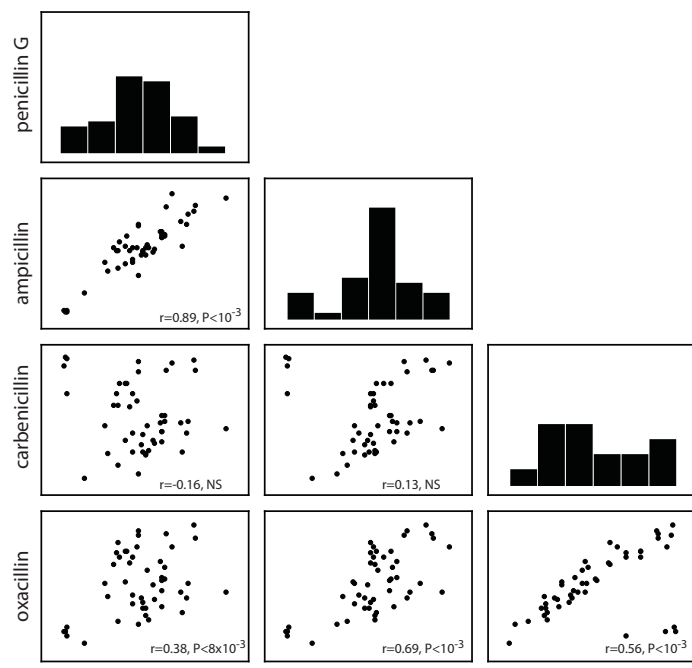

ampicillin

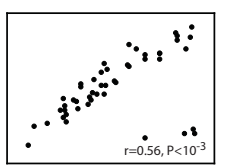

carbenicillin

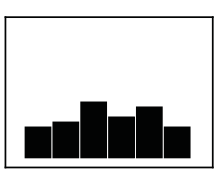

oxacillin

Fig. (4). Covariation of activity on four different antibiotics after selection for activity on ampicillin. All data in this figure are based on 48 clones from TEM-1 populations subject to mutagenesis and subsequent selection for activity on the antibiotic ampicillin (Fig. 1). The four histograms in the diagonal of the lower-diagonal matrix indicate, from top to bottom, the distribution of activities of these clones on penicillin G, ampicillin, carbenicillin, and oxacillin. The scatterplots indicate activities on pairs of antibiotics, as indicated to the left of each row and on the bottom of each column. NS: not significant. Axes scales are omitted for clarity and correspond to the following intervals bounded by minimal and maximal measured activity values: $(182.3,1339.2)$ on penicillin $G,(280.0,2325.8)$ on ampicillin, (52.0, 221.2) on carbenicillin, $(18.1,108.3)$ on oxacillin.

are as a whole. To answer this question, we applied common principal component analysis (CPCA, see Methods) to phenotypic covariances that we computed from our five populations (mutagenized-only, mutagenized and selected on A, C, $\mathrm{P}$, and $\mathrm{O})$. CPCA allows us to ask whether all principal components of two or more covariance matrices are equal, whether these principal components are unequal but proportional to one another, and whether the matrices share any number of principal components. The answer to all three questions was no $\left(p<10^{-4}\right)$. This means that the five covariance matrices as a group are unrelated. We then carried out four additional CPCAs, each for a pair of matrices, where we paired the matrix from a selected population with that from the mutagenized population, and did so for the populations selected on A,C, P, and O. For each of these pairs, and for each of the hypotheses of shared matrix structure, CPCA rejected the hypothesis at $p<10^{-4}$. This means that selection alters the covariation of activity on the four antibiotics, such that it becomes statistically unrelated to covariation after mutagenesis. Finally, we also compared covariance matrices for those selection lines that shared the greatest number of significant correlations (carbenicillin and penicillin, Fig. 5C and $5 \mathbf{E}$ ). Again, CPCA rejected any of the possible hypotheses of shared matrix structure. In sum, according to CPCA, the patterns of activity covariation that we observe appear statistically unrelated among mutagenized and selected lines. 


\section{Most Phenotypic Variation we Observe Reflects Genetic Variation}

Phenotypic variation in activity among TEM-1 clones has two principal sources. The first is genetic differences in the clones caused by mutagenesis. The second comprises the inevitable environmental differences that clones experience as they grow from single cells, and as these cells are prepared for activity measurements. Cast in the language of quantitative genetics, the phenotypic variance $V_{P}$ in activity is composed of a genetic variance $V_{G}$ and an environmental variance $V_{E}$. In the absence of genotype-by-environment interactions, $V_{P}=V_{G}+V_{E}$ [70]. From an evolutionary standpoint, the genetic variance is especially important, because it is mostly genetic variation that is inherited from generation to generation, especially in a system as simple as our study protein.
All variation in activity measurements that we reported so far reflect phenotypic variation, and not necessarily genetic variation. To find out which part of phenotypic variation reflects genetic variation one can carry out an analysis of variance [70, Chapter 18], which requires multiple replicate activity measurements of each clone. While such replication would be too time-consuming for all our selection lines, we carried it out for one of our selected populations and for a subset of two antibiotics. Specifically, we carried out five replicate measurements of activity on ampicillin for each of 48 clones of the population selected on ampicillin (i.e., a total of 240 measurements). We found that the vast majority of the contribution to the phenotypic variance $\left(V_{P} \approx 2.19 \times 10^{5}\right)$ comes from the genetic variance $\left(V_{G} \approx 2.04 \times 10^{5}\right)$, and only a minor part from the environmental variance $\left(V_{E} \approx 1.5 \times 10^{4}\right)$. These two variance components allow us to estimate the broad-sense heritability as $H^{2}=V_{G} / V_{T} \approx 0.93$, meaning that 93

A) Mutagenesis only

\begin{tabular}{l|llll} 
& penicillin G & ampicillin & carbenicillin & oxacillin \\
\hline penicillin G & 40822 & 72374 & 1143 & 3273 \\
ampicillin & 0.99 & 129702 & 2034 & 5871 \\
carbenicillin & 0.96 & 0.96 & 35 & 96 \\
oxacillin & 0.97 & 0.98 & 0.98 & 277
\end{tabular}

B)

Selection on ampicillin

\begin{tabular}{l|llll} 
& penicillin G & ampicillin & carbenicillin & oxacillin \\
penicillin G & 63533 & 106736 & -1885 & 2280 \\
ampicillin & 0.89 & 228595 & 2843 & 7904 \\
carbenicillin & -0.16 & 0.13 & 2239 & 633 \\
oxacillin & 0.38 & 0.69 & 0.56 & 578
\end{tabular}

C) Selection on carbenicillin

\begin{tabular}{l|llll}
\multicolumn{1}{c}{} & penicillin G & ampicillin & carbenicillin & oxacillin \\
\hline penicillin G & 159338 & 131622 & 4225 & 1513 \\
ampicillin & 0.99 & 111579 & 3555 & 1212 \\
carbenicillin & 0.5 & 0.5 & 448 & 192 \\
oxacillin & 0.38 & 0.36 & 0.9 & 101
\end{tabular}

D) Selection on oxacillin

\begin{tabular}{l|llll}
\multicolumn{1}{c}{} & penicillin G & ampicillin & carbenicillin & oxacillin \\
\hline penicillin G & 38596 & 64045 & 338 & -164 \\
ampicillin & 0.93 & 121984 & 589 & -164 \\
carbenicillin & 0.07 & 0.06 & 687 & 293 \\
oxacillin & -0.07 & -0.04 & 0.92 & 148
\end{tabular}

\begin{tabular}{l|llll}
\multicolumn{1}{c}{} & penicillin G & ampicillin & carbenicillin & oxacillin \\
\hline penicillin G & 112184 & 162602 & 15940 & 5407 \\
ampicillin & 0.98 & 243885 & 22831 & 7824 \\
carbenicillin & 0.88 & 0.86 & 2916 & 923 \\
oxacillin & 0.89 & 0.87 & 0.93 & 331
\end{tabular}

Fig. (5). Variance, covariance, and Pearson correlation coefficients of activities on four different antibotics. The values shown in the upper triagonal of each matrix show the phenotypic covariance $\sigma_{i j}{ }^{2}$ of hydrolytic activities on antibiotics $i$ and $j$, as listed to the left of each matrix row, and on the top of each matrix column. The diagonal element show the variances $\sigma_{i}^{2}$ of hydrolytic activity on antibiotic $i$. The values in the lower diagonal elements are Pearson product-moment correlation coefficients $r$ of hydrolytic activities. Correlation coefficients in black type are significantly different from zero at $p<0.01$. All data are based on 48 TEM- 1 variants, as described in the text. A) activity measurements from mutagenized TEM-1 populations without selection. Values in B-E are from mutagenized TEM-1 populations selected for activity on B) ampicillin, C) carbenicillin, D) oxacillin, and E) penicillin G. 
percent of the phenotypic variance is contributed by genetic variance. (Note that the narrow-sense heritability, an important, related concept in quantitative genetics, is meaningless for clonal populations like ours [70].) We also wanted to make sure that this high heritability is not just an artifact of having measured activity on the same antibiotic we selected for. To this end, we also measured activity on carbenicillin of the same ampicillin-selected clones in quintuplicate. Here, the environmental variance $\left(V_{E}=41.2\right)$ constituted an even smaller fraction of the phenotypic variance $\left(V_{P}=2007.9\right)$, yielding a very high heritability of $H^{2}=V_{G} / V_{T} \approx 0.98$. In sum, these two observations show that the vast majority of the variance in activity reflects heritable genetic variation. If true for activities on all antibiotics that we considered, this means that the phenotypic correlations we observe (Figs 3, 4, and 5) largely reflect genetic correlations, which are not obscured by environmental variation.

\section{Phenotypic Hitchhiking}

In our experiments, selection on the antibiotic ampicillin was most effective in increasing activity on this antibiotic. Given that activities covary positively for different antibiotics in our data, this effectiveness may also translate into activity increases on other antibiotics. This is indeed the case. More than that, in some of our selected populations, selection on one antibiotic A can lead to larger absolute increases in activity on antibiotic B than selection on antibiotic B itself. For brevity, we refer to this phenomenon as phenotypic hitchhiking, because activity on antibiotic B benefits indirectly through an increase in activity on antibiotic A. For example, in the population selected on ampicillin, the mean activity on penicillin $G$ was greater than in the population selected on penicillin $\mathrm{G}$ itself ( $p=0.0063$, Mann-Whitney Utest , Fig. 6A). The same holds for the activities on carbenicillin and oxacillin. They are significantly higher in the ampicillin-selected population than in the populations selected on carbenicillin $\left(p=10^{-13}\right.$, Mann-Whitney U-test, Fig. 6B) and oxacillin ( $p=1.6 \times 10^{-7}$, Mann-Whitney U-test, Fig. 6C), respectively. The most dramatic of these differences relative to the mutagenized population occurs with respect to the antibiotic carbenicillin. Here, selection on carbenicillin increased the mean activity by more than 4.6 fold to $64.3 \pm 21.2$ relative to the mutagenized population (13.7 \pm 5.9 , Fig. 2A), whereas selection on ampicillin increased activity on carbenicillin more than 10 -fold to a level of $138.9 \pm 47.3$. This activity is somewhat higher than in the ancestor (123.6 \pm 6.9$)$, although the difference to the ancestor in activity is not significant $(p=0.77)$. Activities on the other two antibiotics were also not significantly higher (oxacillin), or they were slightly lower (penicillin $G$ ) than in the ancestor.

Phenotypic hitchhiking is not just restricted to the population selected on ampicillin. For example, selection on penicillin increases activity on carbenicillin more than selection on carbenicillin itself $\left(p=1.3 \times 10^{-7}\right.$, Mann-Whitney U-test , Fig. 6D), even though it was not successful in raising activity on penicillin above the ancestor. The same holds for selection on oxacillin, which increases activity on carbenicillin to a greater extent than selection on carbenicillin itself $\left(p=10^{-5}\right.$, Mann-Whitney U-test, Fig. 6E).

Phenotypic hitchhiking is easy to explain through basic quantitative genetics theory [70, 71]. Quantitative genetics describes the change in the population mean of a multivariate trait $\mathbf{z}$ as $\Delta \overline{\mathbf{z}}=\mathbf{G} \boldsymbol{\beta}$, where $\Delta \overline{\mathbf{z}}=\left(\Delta \bar{z}_{1}, \ldots, \Delta \bar{z}_{n}\right)$ is the change in the means of univariate traits 1 through $n$ after selection, $\mathbf{G}$ is a matrix of genetic covariances, and $\boldsymbol{\beta}=\left(\beta_{1}, \ldots, \beta_{n}\right)$ the selection gradient, a vector of partial regression coefficients $\beta_{i}$ of fitness on trait $i$ [71]. To see the reason why phenotypic hitchhiking occurs, consider the example of two hypothetical traits 1 and 2, where trait 1 has some genotypic value with mean $\mu_{1}$ and standard deviation $\sigma_{1}$. Trait 2 has a greater mean and standard deviation, say equal to $\mu_{2}=10 \mu_{1}$ and $\sigma_{2}=10 \sigma_{2}$. If traits 1 and 2 are positively correlated, e.g., with a genetic correlation of $r_{12}=\frac{\sigma_{12}}{\sigma_{1} \sigma_{2}}=0.5$, then the covariance matrix $\mathbf{G}$ becomes

$$
\mathbf{G}=\left(\begin{array}{cc}
\sigma_{1}^{2} & 5 \sigma_{1}^{2} \\
5 \sigma_{1}^{2} & 100 \sigma_{1}^{2}
\end{array}\right)
$$

We can now ask how trait 1 responds if selection occurs predominantly on trait 1 or on trait 2 . For example, if $\boldsymbol{\beta}=(2 \beta, \beta)$, such that selection acts more strongly on trait 1 , then $\Delta \overline{\mathbf{z}}=\mathbf{G} \boldsymbol{\beta}=\left(7 \beta \sigma_{1}^{2}, 110 \beta \sigma_{1}^{2}\right)$. Thus, the mean of trait 1 increases by $7 \beta \sigma_{1}^{2}$ units. Conversely, if selection acts more strongly on trait 2, e.g., $\boldsymbol{\beta}=(\beta, 2 \beta)$, the $\Delta \overline{\mathbf{z}}=\mathbf{G} \boldsymbol{\beta}=\left(11 \beta \sigma_{1}^{2}, 205 \beta \sigma_{1}^{2}\right)$, and the mean of trait 1 increases by $11 \beta \sigma_{1}^{2}$ units. Thus, when selection acts preferentially on trait 1 , selection increases the mean of trait 1 by a smaller amount than if selection acts directly on trait 2 . More generally, it is easy to show in this framework that phenotypic hitchhiking will occur if (i) $\sigma_{2}^{2}>\sigma_{1}^{2}$, and if (ii) the genetic covariance $\sigma_{12}$ is greater than the genetic variance $\sigma_{1}^{2}$ of trait one. Phenotypic hitchhiking is a consequence of indirect selection that arises in covariance matrices with these features. One can also view it as a result of the fact that different traits have different means and thus usually also different variances. Although our experiments strictly speaking do not reveal the structure of the genotypic covariance matrix, we note that the phenotypic covariance matrices of our traits (Fig. 5) have the required structure. For example, the covariance between activity on penicillin $\mathrm{G}$ and ampicillin is greater than the variance of activity on penicillin $G$, but smaller than the variance of activity on ampicillin (Fig. 5). These are the conditions where activity on penicillin $G$ can hitchhike on selection for ampicillin activity, exactly as we observe (Fig. 6A).

\section{DISCUSSION AND CONCLUSION}

We analyzed to which extent mutagenesis of the TEM-1 $\beta$-lactamase alone, or mutagenesis followed by selection on four different antibiotics, affects the hydrolytic activity of this enzyme. Mutagenesis alone significantly reduced activity on all four antibiotics. Subsequent selection partially or fully recovered activity on all antibiotics, but changed the extent to which these activities covary. Specifically, mutagenesis alone caused highly positively correlated activities on the four focal antibiotics, but subsequent selection re- 
duced these correlations, and rendered the activities on several antibiotics uncorrelated. (Not a single pair of antibiotics showed a significantly negative activity correlation.) The phenotypic covariance matrices of activity on different antibiotics were statistically unrelated among our five populations. We also observed several instances where selection on one antibiotic A can increase the activity of another antibiotic B to a greater extent than selection on antibiotic B itself.

Phenotypic and genetic covariation among traits limit what natural selection can achieve, because selection cannot shape two covarying traits independently. Typically, genetic studies of trait covariation compare complex morphological or physiological traits of multicellular organisms [20, 21, 2729, 44-47, 72]. To our knowledge, analyses of trait covariation have not yet been extensively applied to much simpler, molecular systems like individual proteins, although measurements of more than one activity in modest numbers of proteins are not unusual in protein engineering [18, 49, 73-77]. The advantage that molecular systems like proteins have over organisms in the wild is that they can be easily manipulated in the laboratory through mutagenesis and different kinds of selection pressure.

One frequently asked question about macroscopic traits is whether patterns of trait covariation themselves are subject

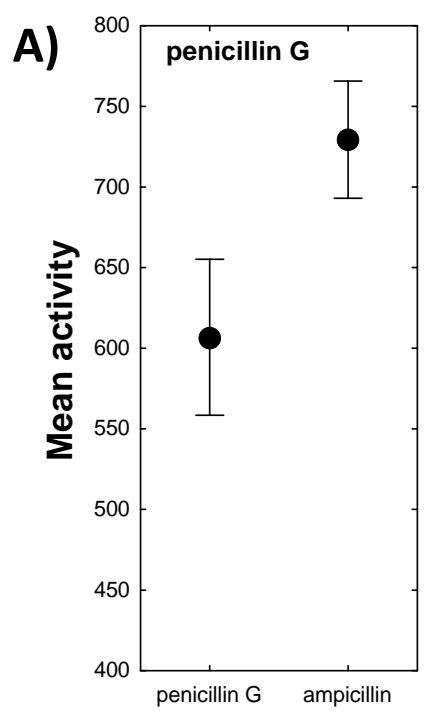

Selection

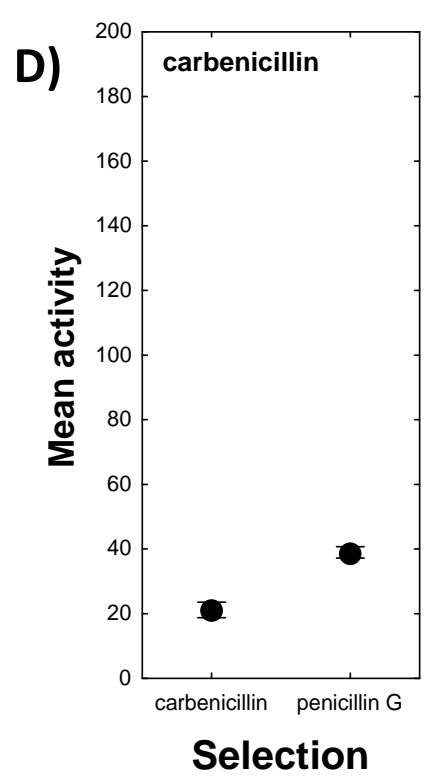

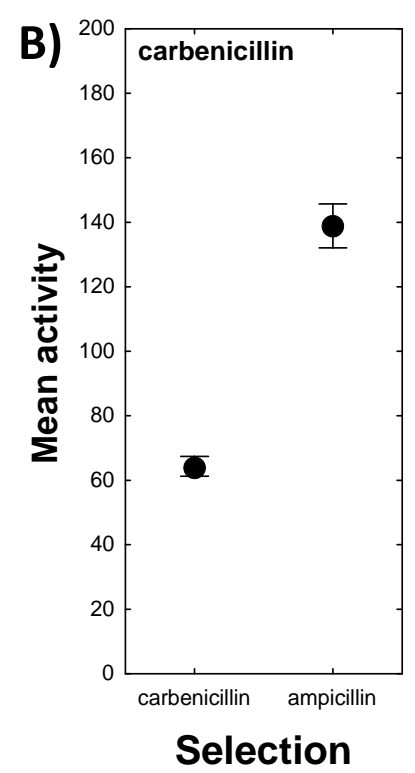
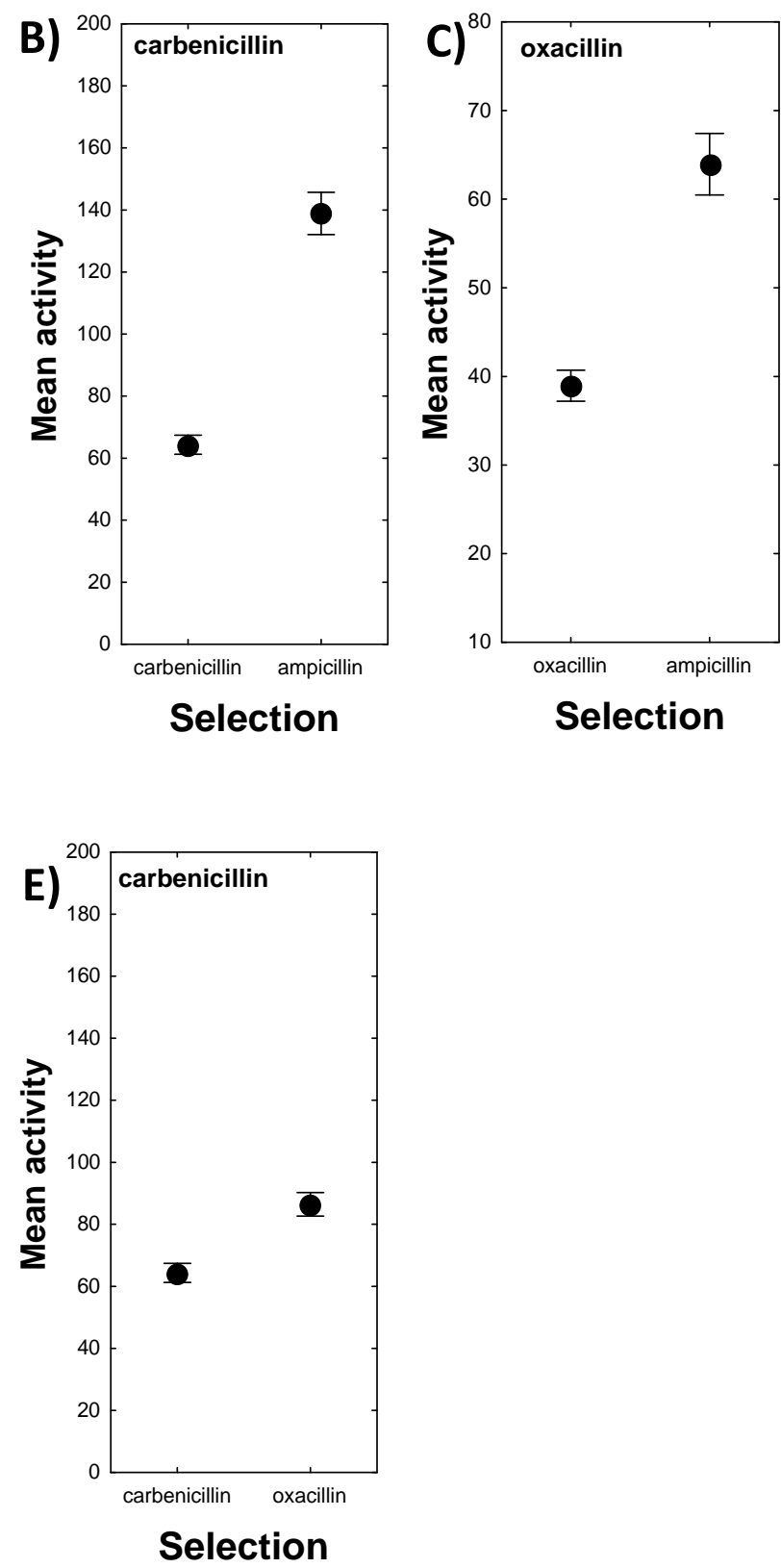

Fig. (6). Phenotypic hitchhiking of activities on an antibiotic. The vertical axis of each panel indicates the average (dot) and one standard error of the mean (whiskers) activity of 48 TEM-1 derived clones on the antibiotic whose known is shown in the inset. These clones had been subjected to mutagenesis followed by selection for activity on the antibiotics indicated on the x-axis. A) activity on penicillin $\mathrm{G}$ for lines selected on penicillin $\mathrm{G}$ and ampicillin; B) activity on carbenicillin for lines selected on carbenicillin and ampicillin; C) activity on oxacillin for lines selected on oxacillin and ampicillin; D) activity on carbenicillin for lines selected on carbenicillin and penicillin G; E) activity on carbenicillin for populations selected on carbenicillin and oxacillin. Units are mODU $\left(\mathrm{min}^{-1}\right)(10 \mu l \text { extract })^{-1}$. 
to evolutionary change. The answer depends strongly on the study species, their phylogenetic diversity, and on the traits studied. It ranges from completely conserved to completely diverged covariation structure [20, 27, 28, 43-47, 72]. Pertinent studies often involve species that have diverged many generations ago, and through incompletely understood evolutionary forces. In contrast, a simple study system like ours is ideally suited to ask how covariation patterns evolve on very short time scales, and under precisely controllable conditions.

Our observations show that even a single round of selection can substantially alter phenotypic covariation patterns. On the one hand, mutagenesis alone resulted in a significant decrease in TEM-1 activity on all four antibiotics. This decrease was highly correlated among different antibiotics, with a correlation coefficient exceeding $r=0.95$ for each antibiotic pair (Fig. 5A). On the other hand, selection substantially changed some of these correlations. For example, the correlation in activity between ampicillin and oxacillin was reduced from $r=0.98$ in the mutagenized population to $r=0.87$ and $r=0.69$ in the populations selected on penicillin $G$ and on ampicillin, respectively. The correlation became indistinguishable from zero in populations selected on carbenicillin and oxacillin (Fig. 5). The extent of this reduction does not only depend on the selection pressure applied, but also on the antibiotic pair. For example, while activity correlations between ampicillin and penicillin $G$ were high $(r>0.88)$ in populations selected on ampicillin and oxacillin (and in all other populations as well), those between ampicillin and carbenicillin became insignificant after selection on these two antibiotics. Common principal component analysis [60, 64] showed that the covariance matrices of activities on the four different antibiotics have little similarity. These observations show that selection can rapidly change trait covariances and correlation. We caution that we only estimated the phenotypic component of covariation among traits, but not its genetic component. Estimation of the latter is much more laborious, even though phenotypic covariation is often a good reflection of genetic covariation [27]. We did, however, demonstrate a very high broad-sense heritability of activity on carbenicillin and ampicillin $\left(H^{2}>0.92\right)$, showing that the vast majority of the variance in these activities is genetic variance. If the same holds for the other activities we analyzed, then the patterns of change in phenotypic covariance we observe reflect changes in genetic covariance.

One might argue that a reduction in genetic variation caused by selection may be partly responsible for the smaller phenotypic correlations we see between many traits after selection. Specifically, selection might reduce the genetic variance in a trait caused by mutagenesis, such that environmental variance becomes relatively more important. This environmental variance might obscure genetic correlations among our traits, leading to smaller phenotypic correlations. We note that our very high estimates of heritability after selection argue against this possibility.

In contrast to more complex traits, the simple traits we study may allow at least some insight into the proximate reason between trait covariation. Fig. (7), for example, shows the chemical structures of the four antibiotics used in this study, where parts of the molecules in common between all four antibiotics are shaded in gray. The Figure illustrates that ampicillin is highly similar to penicillin $\mathrm{G}$ - the two differ in a single amino group - but quite different from oxacillin. The high similarity between ampicillin and penicillin $\mathrm{G}$ may explain why activity correlations between them are uniformly high in all five populations. In contrast, the correlation between activity on ampicillin and oxacillin is much more variable among the five enzyme populations we studied. Specifically, depending on the selection pressure applied, these correlations can assume values different from one another, where one may be significantly different from zero, whereas the other is not. These differences may reflect the greater chemical differences between these antibiotics. However, chemical similarity or dissimilarity may not explain all of the differences in covariation patterns we observe. For example, carbenicillin is also quite similar to ampicillin, and differs from it only in a carboxyl group. Nonetheless, selection is much less effective in increasing activity on carbenicillin than on ampicillin (Fig. 2A and Fig. 2D). Also, the covariation between activity on ampicillin and carbenicillin is no less variable than the covariation between the much more diverse oxacillin and ampicillin (Fig. 5). Ultimately, to understand patterns like this we would need to know how $\beta$-lactamase interacts with different antibiotics on an atomic scale, and how different $\beta$-lactamase mutants differ in this interaction, as well as in other properties, such as their thermodynamic stability. For other proteins, a combination of experimental and computational work is beginning to facilitate understanding of how biophysical and chemical properties like these can affect the molecular evolution of proteins [78-85]. But even though $\beta$-lactamases are extremely well-studied biochemically and evolutionarily, their interactions with antibiotics are still insufficiently understood. Recent progress is being made in this area [86-88]. If it continues, we will be able to link the biophysics and biochemistry of these enzymes to the population-wide patterns we observed.

Trade-offs between traits are not only important to evolutionary biologists [37-42], but also to protein engineers, as they can severely limit possibilities to improve any one trait. Available evidence for such trade-offs in proteins typically involve few (as little as two) mutant proteins and their activity on two different substrates [18, 73-77]. If one mutant has a high activity on substrate $A$ and a low activity on substrate $\mathrm{B}$, whereas another mutant shows the opposite relationship, a trade-off may exist. However, to infer trade-offs based on few variants has serious limitations, as the example of Fig. (8) illustrates. The figure shows the activities on penicillin $G$ and oxacillin of 48 clones selected on oxacillin. The vertical and horizontal solid lines show the activities of the TEM-1 ancestor. There are several clones with higher than ancestral activity on oxacillin, and lower than ancestral activity on penicillin $\mathrm{G}$ (upper left quadrant), or vice versa (lower right quadrant). Comparison of individual such clones to the wild-type could be construed as evidence for a trade-off. However, the overall association between the activities is not significantly different from zero $(\mathrm{r}=-0.07)$, and no evidence of a trade-off exist. This simple example shows that unless a number of variants high enough for statistical analysis is examined, solid evidence for a trade-off is hard to come by. In our five study population, no two antibiotic activities show a trade-off. The reason may lie in the chemical similarity of the antibiotics we studied, as more distantly related antibiotics, such as cefotaxime or ceftazidime with bulkier side chains may display such trade-offs [74]. 


\section{Ampicillin}<smiles>CC1(C)SC2C(NC(=O)C(N)c3ccccc3)C(=O)N2C1C(=O)O</smiles>

Carbenicillin

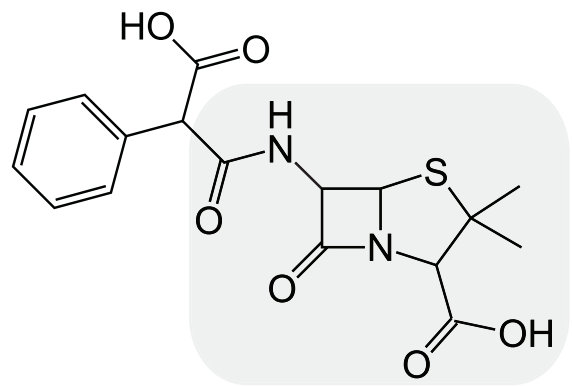

\section{Penicillin G}
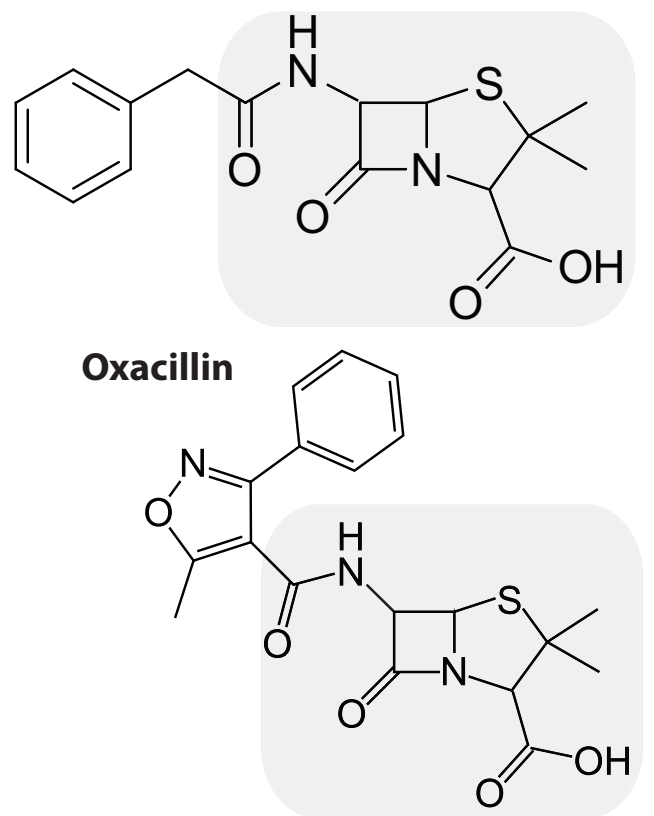

Fig. (7). Chemical structure of the four antibiotics used in this study. The shaded area includes groups shared by all four antibiotics.

Our observation of phenotypic hitchhiking implies that to increase activity on a substrate A, it may not always be best to select for activity on A itself, but instead on a related substrate B where activity covaries positively with activity on A. We observed phenotypic hitchhiking for selection on ampicillin, where selection increased activity on $\mathrm{P}, \mathrm{O}$, or $\mathrm{C}$ to a greater extent than selection on $\mathrm{P}, \mathrm{O}$, or $\mathrm{C}$ itself (Fig. 6A6C). We also observed it, to a lesser extent, for selection on penicillin $\mathrm{G}$ and oxacillin (Fig. 6D and $\mathbf{E}$ ). Basic quantitative genetic theory implies that phenotypic hitchhiking occurs when the genotypic variances $\left(\sigma_{1}^{2}, \sigma_{2}^{2}\right)$ and covariance $\left(\sigma_{12}\right)$ of activity on substrate 1 and 2 follow the order relation $\sigma_{1}^{2}<\sigma_{12<} \sigma_{2}^{2}$. Phenotypic hitchhiking can thus be viewed as a simple consequence of the fact that different, positively covarying traits, have different variances. For some applications, such as to compare the relative changes in traits that selection can achieve, it may be best to rescale and standardize trait means or variances [29, 89]. However, wherever absolute changes in a trait matter most, for example in many biotechnological applications, such rescaling may not be appropriate.

The extent of phenotypic hitchhiking will depend on the extent to which the variances and covariances of two traits differ. For example, in our experiments phenotypic hitchhiking was most effective involving selection on ampicillin (Fig. 6A-6C), because ampicillin activity shows a much greater variance than other activities, as well as a high positive covariance with the other three traits (Fig. 5). (Although the (co)variances we observe are phenotypic and not genotypic co-variances, the fact that our analysis is based on clonal populations, and our partial analysis of heritability suggest that the two kinds of (co)variances will be similar.) Phenotypic hitchhiking may have practical applications. For example, if one desires to improve the activity of a promiscuous enzyme on some substrate $\mathrm{B}$, and if directed evolution is known to be able to improve activity on a closely related substrate A, then selection on A may be an effective strategy to increase activity on B.

We view our study as a first step towards applying analyses of trait covariation to molecular systems, and are well aware that it leaves many open questions. How dramatically can selection alter trade covariation over longer time scales, that is, after multiple rounds of selection? Will negative trade-offs between activities arise where none existed before? How well can selection alleviate such trade-offs? How dissimilar do the substrates of an enzyme need to be before negative trade-offs arise? Simple molecular systems may help answer questions about trait covariation that speak to long-standing questions in evolutionary biology which are difficult to address in more complex systems.

\section{ACKNOWLEDGEMENTS}

AW acknowledges support through Swiss National Science Foundation grant 315230-129708, as well as through the YeastX project of SystemsX.ch, and the University Priority Research Program in Systems Biology at the University of Zurich. We would like to thank Gabriel Marroig for comments on a previous version of this manuscript.

\section{CONFLICT OF INTEREST}

The authors confirm that this article content has no conflicts of interest. 


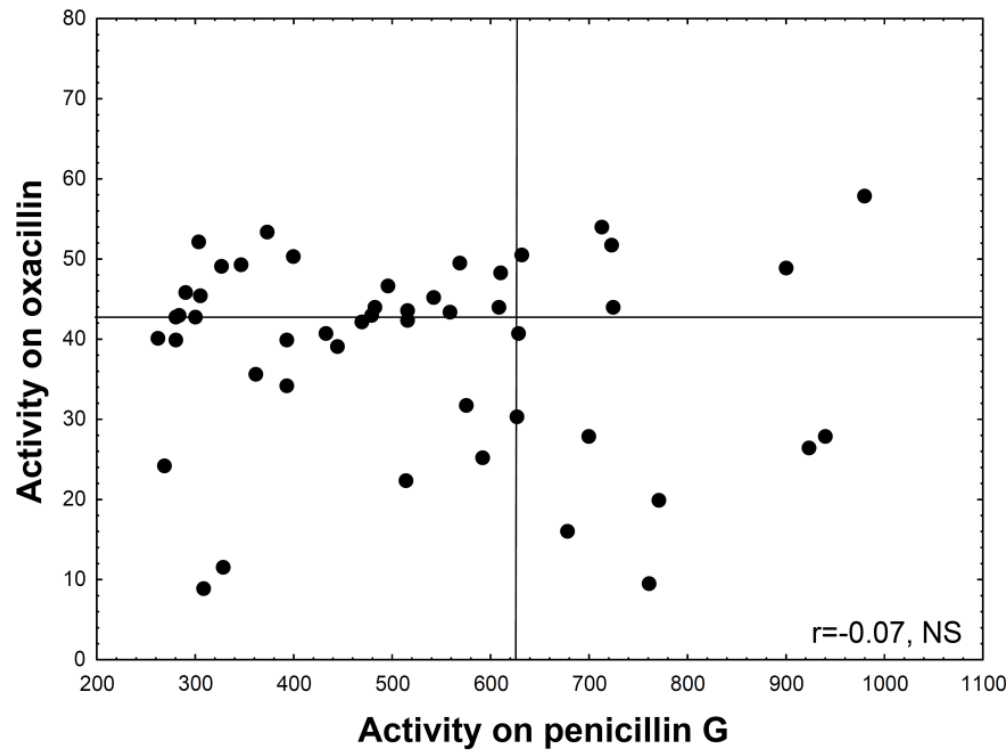

Fig. (8). No trade-off in activity on penicillin $G$ and oxacillin. The data shows activities on penicillin $G$ and oxacillin for 48 clones selected for activity on oxacillin. The solid horizontal line indicates ancestral activity on oxacillin, the vertical solid line ancestral activity on penicillin G. Note that several clones show higher activity than the ancestor on one antibiotic but lower activity on the other antibiotic (points in upper left and lower right quadrant), but no trade-off exists between the activities, because their association is not significantly different from zero $(\mathrm{r}=-0.07, p>0.05)$. Units are mODU $\left(\mathrm{min}^{-1}\right)(10 \mu \mathrm{l} \text { extract })^{-1}$.

\section{REFERENCES}

[1] West-Eberhard, M. Developmental Plasticity and Evolution. Oxford University Press: New York, NY, 2003.

[2] Hölldobler, B.; Wilson, E. O. The Ants. Belknap Press of Harvard University Press: Cambridge, MA, 1990.

[3] Schmalhausen, I. I. Factors of Evolution. Blakiston: Philadelphia, 1949.

[4] Hubel, D. H. Effects of distortion of sensory input on the visual system of kittens. Physiologist, 1967, 10, 17-45.

[5] Gilbert, S. F. Developmental Biology. $5^{\text {th }}$ ed.; Sinauer: Sunderland, 1997.

[6] Rao, C.; Wolf, D.; Arkin, A. Control, exploitation and tolerance of intracellular noise. Nature, 2002, 420, 231-237.

[7] Ozbudak, E.; Thattai, M.; Kurtser, I.; Grossman, A.; van Oudenaarden, A. Regulation of noise in the expression of a single gene. Nat. Genet., 2002, 31, 69-73.

[8] McAdams, H. H.; Arkin, A. It's a noisy business! Genetic regulation at the nanomolar scale. Trends Genet., 1999, 15, 65-69.

[9] Elowitz, M.; Levine, A.; Siggia, E.; Swain, P. Stochastic gene expression in a single cell. Science, 2002, 297, 1183-1186.

[10] Blake, W.; Kaern, M.; Cantor, C.; Collins, J. Noise in eukaryotic gene expression. Nature, 2003, 422, 633-637.

[11] Bar-Even, A.; Paulsson, J.; Maheshri, N.; Carmi, M.; O'Shea, E.; Pilpel, Y.; Barkai, N. Noise in protein expression scales with natural protein abundance. Nat. Genet., 2006, 38, 636-643.

[12] Becskei, A.; Kaufmann, B. B.; van Oudenaarden, A. Contributions of low molecule number and chromosomal positioning to stochastic gene expression. Nat. Genet., 2005, 37, 937-944.

[13] Becskei, A.; Serrano, L. Engineering stability in gene networks by autoregulation. Nature, 2000, 405, 590-593.

[14] Eldar, A.; Elowitz, M. B. Functional roles for noise in genetic circuits. Nature, 2010, 467, 167-173.

[15] Jeffery, C. Moonlighting proteins. Trends Biochem. Sci., 1999, 24, 8-11.

[16] O'Brien, P. J.; Herschlag, D. Catalytic promiscuity and the evolution of new enzymatic activities. Chem. Biol., 1999, 6, R91-R105.

[17] Hult, K.; Berglund, P. Enzyme promiscuity, mechanism and applications. Trends Biotechnol., 2007, 25, 231-238.
[18] Khersonsky, O.; Tawfik, D. S. Enzyme promiscuity, A mechanistic and evolutionary perspective. Ann. Rev. Biochem., 79, 471-505.

[19] Vitreschak, A. G.; Rodionov, D. A.; Mironov, A. A.; Gelfand, M. S. Riboswitches, the oldest mechanism for the regulation of gene expression? Trends Genet., 2004, 20, 44-50.

[20] Dodd, R. S.; Rafii, Z. A.; Bousquet-Melou, A. Evolutionary divergence in the pan-Atlantic mangrove Avicennia germinans. New Phytol., 2000, 145, 115-125.

[21] Steppan, S. J. Phylogenetic analysis of phenotypic covariance structure .1. Contrasting results from matrix correlation and common principal component analyses. Evolution, 1997, 51, 571-586.

[22] Futuyma, D. J. Evolutionary Biology. $3^{\text {rd }}$ ed. Sinauer: Sunderland, Massachusetts, 1998.

[23] Atchley, W. R.; Rutledge, J. J.; Cowley, D. E. Genetic components of size and shape .2. Multivariate covariance patterns in the rat and mouse skull. Evolution, 1981, 35, 1037-1055.

[24] Goodin, J. T.; Johnson, M. S. Patterns of morphological covariation in Partula. Syst. Biol., 1992, 41, 292-304.

[25] Arnold, S. J. Constraints on phenotypic evolution. Am Nat.,1992, 140, S85-S107.

[26] Schluter, D. Adaptive radiation along genetic lines of least resistance. Evolution, 1996, 50, 1766-1774.

[27] Steppan, S. J.; Phillips, P. C.; Houle, D. Comparative quantitative genetics, evolution of the G matrix. Trends Ecol. Evol., 2002, 17, 320-327.

[28] Game, E. T.; Caley, M. J. The stability of P in coral reef fishes. Evolution, 2006, 60, 814-823.

[29] Hansen, T. F.; Houle, D. Measuring and comparing evolvability and constraint in multivariate characters. J. Evol. Biol., 2008, 21, 1201-1219.

[30] Amundson, R. 2 Concepts of constraint - adaptationism and the challenge from developmental biology. Philos. Sci., 1994, 61, 556578.

[31] Antonovics, J.; Vantienderen, P. H. Ontoecogenophyloconstraints the chaos of constraint terminology. Trends Ecol Evol., 1991, 6, 166-168.

[32] Brakefield, P. M. Evo-devo and constraints on selection. Trends Ecol. Evol., 2006, 21, 362-368.

[33] Hodin, J. Plasticity and constraints in development and evolution. In: Modularity of Animal Form Workshop, Friday Harbor, Washington, Friday Harbor: Washington D.C. 2000, pp. 1-20 
[34] Maynard-Smith, J.; Burian, R.; Kauffman, S.; Alberch, P.; Campbell, J.; Goodwin, B.; Lande, R.; Raup, D.; Wolpert, L. Developmental constraints and evolution. Q. Rev. Biol., 1985, 60, 265-287.

[35] Wagner, A. Evolutionary constraints permeate metabolic networks. BMC Evol. Biol., 2009, 9, 231.

[36] Alberch, P.; Gale, E. A. A developmental analysis of an evolutionary trend - digital reduction in amphibians. Evolution, 1985, 39, 823.

[37] Pfeiffer, T.; Schuster, S.; Bonhoeffer, S. Cooperation and competition in the evolution of ATP-producing pathways. Science, 2001, 292, 504-507.

[38] Charnov, E. L. Trade-off-invariant rules for evolutionarily stable life-histories. Nature, 1997, 387, 393-394.

[39] Carr, D. E.; Murphy, J. F.; Eubanks, M. D. Genetic variation and covariation for resistance and tolerance to Cucumber mosaic virus in Mimulus guttatus (Phrymaceae), a test for costs and constraints. Heredity, 2006, 96, 29-38.

[40] Martin, O. C.; Wagner, A. Multifunctionality and robustness tradeoffs in model genetic circuits. Biophys J., 2008, 94, 2927-2937.

[41] Miller, S. P.; Lunzer, M.; Dean, A. M. Direct demonstration of an adaptive constraint. Science, 2006, 314, 458-461.

[42] Bennett, A. F.; Lenski, R. E. Evolutionary adaptation to temperature .2. Thermal niches of experimental lines of Escherichia coli. Evolution, 1993, 47, 1-12.

[43] Franks, S. J.; Weis, A. E. A change in climate causes rapid evolution of multiple life-history traits and their interactions in an annual plant. J. Evol. Biol., 2008, 21, 1321-1334.

[44] Christensen, K. I. A morphometric study of the geographic variation in Pinus contorta (Pinaceae). Nord. J. Bot., 2005, 23, 563-575.

[45] Badyaev, A. V.; Hill, G. E. The evolution of sexual dimorphism in the house finch. I. Population divergence in morphological covariance structure. Evolution, 2000, 54, 1784-1794.

[46] Ackermann, R. R.; Cheverud, J. M. Phenotypic covariance structure in tamarins (genus Saguinus), A comparison of variation patterns using matrix correlation and common principal component analysis. Am J. Phys. Anthropol., 2000, 111, 489-501.

[47] Arnold, S. J.; Phillips, P. C. Hierarchical comparison of genetic variance-covariance matrices. II. Coastal-inland divergence in the garter snake, Thamnophis elegans. Evolution, 1999, 53, 1516-1527.

[48] Bloom, J.; Romero, P.; Lu, Z.; Arnold, F. Neutral genetic drift can alter promiscuous protein functions, potentially aiding functional evolution. Biol. Direct, 2007, 2, 17.

[49] Aharoni, A.; Gaidukov, L.; Khersonsky, O.; Gould, S. M.; Roodveldt, C.; Tawfik, D. S. The 'evolvability' of promiscuous protein functions. Nat. Genet., 2005, 37, 73-76.

[50] Livermore, D. M. Beta lactamases in laboratory and clinical resistance. Clin. Microbiol. Rev., 1995, 8, 557-584.

[51] Matagne, A.; Lamotte-Brasseur, J.; Frere, J. M. Catalytic properties of class A beta-lactamases, efficiency and diversity. Biochem. J., 1998, 330, 581-598.

[52] Rahal, K.; Gerbaud, G. B.; Chabbert, Y. A. Characterization d'un facteur de resistance transferable chez Vibrio cholerae biotype el tor. Ann. Inst. Pasteur, 1973, 124B, 245-247.

[53] Zaslaver, A.; Bren, A.; Ronen, M.; Itzkovitz, S.; Kikoin, I.; Shavit, S.; Liebermeister, W.; Surette, M. G.; Alon, U. A comprehensive library of fluorescent transcriptional reporters for Escherichia coli. Nat. Method., 2006, 3, 623-628.

[54] Kawe, M.; Horn, U.; Pluckthun, A. Facile promoter deletion in Escherichia coli in response to leaky expression of very robust and benign proteins from common expression vectors. Microb. Cell Fact., 2009, 8.

[55] Hanahan, D. Studies on transformation of Escherichia coli with plasmids. J Mol. Biol., 1983, 166, 557-580.

[56] Inoue, H.; Nojima, H.; Okayama, H. High-efficiency transformation of Escherichia coli with plasmids. Gene, 1990, 96, 23-28.

[57] Cadwell, R. C.; Joyce, G. F. Randomization of genes by PCR mutagenesis. Genome Res., 1992, 2, 28-33.

[58] Sykes, R. B.; Bonner, D. P.; Bush, K.; Georgopapadakou, N. H. Azthreonam (SQ 26,776) a synthetic monobactam specifically active against aerobic gram-negative bacteria. Antimicrob. Agents Chemother., 1982, 21, 85-92.

[59] Sykes, R. B.; Nordstrom, K. Microiodometric determination of beta-lactamase activity. Antimicrob. Agents Chemother., 1972, 1, 94-99.
[60] Phillips, P. C.; Arnold, S. J. Hierarchical comparison of genetic variance-covariance matrices. I. Using the Flury hierarchy. Evolution, 1999, 53, 1506-1515.

[61] Arnold, S. J. Behavioral variation in natural populations. 1. Phenotypic, genetic, and environmental correlations between chemoreceptive responses to prey in the garter snake, Thamnophis elegans. Evolution, 1981, 35, 489-509.

[62] Krzanowski, W. J. Between-groups comparison of principal components. J. Am. Stat. Assoc., 1979, 74, 703-707.

[63] Marroig, G.; Melo, D.; Porto, A.; Sebastiao, H.; Garcia, G. Selection response decomposition (srd), a new tool for dissecting differences and similarities between matrices. Evol. Biol., 2011, 38, 225241.

[64] Flury, B. N. Common principal components in k groups. J. Am. Stat. Assoc., 1984, 79, 892-898.

[65] Jolliffe, I. T. Principal Component Analysis. Springer: New York, NY, 2002.

[66] Eyre-Walker, A.; Keightley, P. D. The distribution of fitness effects of new mutations. Nat. Rev. Genet., 2007, 8, 610-618.

[67] Eyre-Walker, A.; Keightley, P. D.; Smith, N. G. C.; Gaffney, D. Quantifying the slightly deleterious mutation model of molecular evolution. Mol. Biol. Evol., 2002, 19, 2142-2149.

[68] Huang, W.; Petrosino, J.; Hirsch, M.; Shenkin, P.; Palzkill, T. Amino acid sequence determinants of beta-lactamase structure and activity. J. Mol. Biol., 1996, 258, 688-703.

[69] Loeb, D.; Swanstrom, R.; Everitt, L.; Manchester, M.; Stamper, S.; Hutchison, C. Complete mutagenesis of the HIV-1 protease. $\mathrm{Na}$ ture, 1989, 340, 397-400.

[70] Lynch, M.; Walsh, B. Genetics And Analysis Of Quantitative Traits. Sinauer: Sunderland, MA, 1998.

[71] Lande, R.; Arnold, S. J. The measurement of selection on correlated characters. Evolution, 1983, 37, 1210-1226.

[72] Steven, J. C.; Delph, L. F.; Brodie, E. D. Sexual dimorphism in the quantitative-genetic architecture of floral, leaf, and allocation traits in Silene latifolia. Evolution, 2007, 61, 42-57.

[73] Delmas, J.; Robin, F.; Carvalho, F.; Mongaret, C.; Bonnet, R. Prediction of the evolution of ceftazidime resistance in extendedspectrum beta-lactamase CTX-M-9. Antimicrob. Agents Chemother., 2006, 50, 731-738.

[74] Wang, X. J.; Minasov, G.; Shoichet, B. K. Evolution of an antibiotic resistance enzyme constrained by stability and activity tradeoffs. J. Mol. Biol., 2002, 320, 85-95.

[75] Schmidt, D. M. Z.; Mundorff, E. C.; Dojka, M.; Bermudez, E.; Ness, J. E.; Govindarajan, S.; Babbitt, P. C.; Minshull, J.; Gerlt, J. A. Evolutionary potential of (beta/alpha)(8)-barrels, Functional promiscuity produced by single substitutions in the enolase superfamily. Biochemistry, 2003, 42, 8387-8393.

[76] Rothman, S. C.; Kirsch, J. F. How does an enzyme evolved in vitro compare to naturally occurring homologs possessing the targeted function? Tyrosine aminotransferase from aspartate aminotransferase. J. Mol. Biol., 2003, 327, 593-608.

[77] Hoffmeister, D.; Yang, J.; Liu, L.; Thorson, J. S. Creation of the first anomeric D/L-sugar kinase by means of directed evolution. Proc Natl. Acad. Sci. USA., 2003, 100, 13184-13189.

[78] Rodrigue, N.; Lartillot, N.; Bryant, D.; Philippe, H. Site interdependence attributed to tertiary structure in amino acid sequence evolution. Gene, 2005, 347, 207-217.

[79] Choi, S. C.; Hobolth, A.; Robinson, D. M.; Kishino, H.; Thorne, J. L. Quantifying the impact of protein tertiary structure on molecular evolution. Mol. Biol. Evol., 2007, 24, 1769-1782.

[80] Goldstein, R. A. The structure of protein evolution and the evolution of protein structure. Curr. Opin. Struct. Biol., 2008, 18, 170177.

[81] Zeldovich, K. B.; Shakhnovich, E. I. Understanding protein evolution, From protein physics to Darwinian selection. Annu. Rev. Phys. Chem., 2008, 59, 105-127.

[82] Yang, J. R.; Zhuang, S. M.; Zhang, J. Impact of translational errorinduced and error-free misfolding on the rate of protein evolution. Mol. Syst. Biol., 2010, 6, 421.

[83] Liberles, D. A.; Tisdell, M. D. M.; Grahnen, J. A. Binding constraints on the evolution of enzymes and signalling proteins, the important role of negative pleiotropy. Proc. Biol. Sci., 2011, 278, 1930-1935. 
[84] Pollock, D. D.; Thiltgen, G.; Goldstein, R. A. Amino acid coevolution induces an evolutionary Stokes shift. Proc. Natl. Acad. Sci.USA., 2012, 109, E1352-E1359.

[85] Liberles, D. A.; Teichmann, S. A.; Bahar, I.; Bastolla, U.; Bloom, J.; Bornberg-Bauer, E.; Colwell, L. J.; de Koning, A. P. J.; Dokholyan, N. V.; Echave, J.; Elofsson, A.; Gerloff, D. L.; Goldstein, R. A.; Grahnen, J. A.; Holder, M. T.; Lakner, C.; Lartillot, N.; Lovell, S. C.; Naylor, G.; Perica, T.; Pollock, D. D.; Pupko, T.; Regan, L.; Roger, A.; Rubinstein, N.; Shakhnovich, E.; Sjolander, K.; Sunyaev, S.; Teufel, A. I.; Thorne, J. L.; Thornton, J. W.; Weinreich, D. M.; Whelan, S. The interface of protein structure, protein biophysics, and molecular evolution. Protein Sci., 2012, 21, 769-785.

[86] Maveyraud, L.; Mourey, L.; Kotra, L. P.; Pedelacq, J. D.; Guillet, V.; Mobashery, S.; Samama, J. P. Structural basis for clinical lon- gevity of carbapenem antibiotics in the face of challenge by the common class A beta-lactamases from the antibiotic-resistant bacteria. J. Am. Chem. Soc., 1998, 120, 9748-9752.

[87] Wang, X. J.; Minasov, G.; Shoichet, B. K. Noncovalent interaction energies in covalent complexes, TEM-1 beta-lactamase and betalactams. Proteins, 2002, 47, 86-96.

[88] Nicola, G.; Tomberg, J.; Pratt, R. F.; Nicholas, R. A.; Davies, C. Crystal Structures of covalent complexes of beta-lactam antibiotics with escherichia coli penicillin-binding protein 5 , toward an understanding of antibiotic specificity. Biochemistry, 2010, 49, 80948104.

[89] Hereford, J.; Hansen, T. F.; Houle, D. Comparing strengths of directional selection, How strong is strong? Evolution, 2004, 58, 2133-2143.

(C) Wagner and Weikert; Licensee Bentham Open.

This is an open access article licensed under the terms of the Creative Commons Attribution Non-Commercial License (http://creativecommons.org/licenses/by-nc/3.0/) which permits unrestricted, non-commercial use, distribution and reproduction in any medium, provided the work is properly cited. 